\title{
On existence of nonassociative LA-ring
}

\section{Inayatur-Rehman and Muhammad Shah and Tariq Shah and Asima Razzaque}

\begin{abstract}
We prove the existence of nonassociative LA-ring and LA-field and discuss some of their special cases. We also give some computational information about them.
\end{abstract}

\section{Introduction}

By definition a left almost ring abbreviated as LA-ring (or AG-ring) is a nonassociative structure having at least two elements together with two binary operations ' + ' and '.'such that $(R,+)$ is an AG-group (LA-group) and $(R,$.$) is$ an AG-groupoid (LA-semigroup) and the two distributive laws also hold in $R$.

Though some work on LA-ring has appeared in print for example see $[5,6,7]$, yet existence of the structure of the nonassociative LA-ring has remained in doubt because all the examples given by different authors so far have associative multiplication.

In this short note we prove the existence of the nonassociative LA-ring by providing its Cayley tables that we have found by Mace 4 and thus we are eventually able to abolish this ambiguity. We also provide examples of some of its special cases. Consequently we hope this note will attract the researchers to the detailed study of LA-rings.

For some useful and fundamental properties of LA-rings, we refer the readers to M. Shah and T. Shah [5]. For example it has been proved in [5] that the

Key Words: LA-ring, AG-ring, left almost field, LA-group, AG-groupoid, existence.

2010 Mathematics Subject Classification: Primary 46G05, 46L05; Secondary 47A30, 47B47.

Received: April, 2011.

Revised: April, 2011.

Accepted: February, 2012. 
condition of left distributivity in the definition of LA-ring with left identity becomes redundant as it follows from right distributivity then. Some application of LA-rings can be seen in $[6,8,9]$. An extension of LA-rings to LA-modules has been studied in T. Shah et al. [7].

We would also like to recall the notion of left almost field. By definition, an LA-ring $(R,+,$.$) with left identity e$ is called left almost field (LA-field) if every nonzero element $a$ of the LA-ring has multiplicative inverse $a^{-1}$ in $R$. We will also prove the existence of the nonassociative LA-field.

\section{Proving existence of nonassociative LA-ring and LA- field by examples}

A trivial example of an LA-ring could be:

(i) Take a nonassociative AG-group $G$ of order $n$ with binary operation + ,

(ii) define multiplication on $G$ as $x \cdot y=0$ where 0 is the left identity $(G,+)$. Then $(G,+,$.$) is an LA-ring.$

A non-trivial LA-ring of smallest possible order is given in Example 1.

Example 1. The following example shows the existence of an LA-ring of order 8 , where

(i) is a nonassociative AG-group,

(ii) is a nonassociative AG-groupoid.

(i)

\begin{tabular}{c|cccccccc}
+ & 0 & 1 & 2 & 3 & 4 & 5 & 6 & 7 \\
\hline 0 & 0 & 1 & 2 & 3 & 4 & 5 & 6 & 7 \\
1 & 2 & 0 & 3 & 1 & 6 & 4 & 7 & 5 \\
2 & 1 & 3 & 0 & 2 & 5 & 7 & 4 & 6 \\
3 & 3 & 2 & 1 & 0 & 7 & 6 & 5 & 4 \\
4 & 4 & 5 & 6 & 7 & 0 & 1 & 2 & 3 \\
5 & 6 & 4 & 7 & 5 & 2 & 0 & 3 & 1 \\
6 & 5 & 7 & 4 & 6 & 1 & 3 & 0 & 2 \\
7 & 7 & 6 & 5 & 4 & 3 & 2 & 1 & 0
\end{tabular}

(ii)

\begin{tabular}{l|llllllll}
$\cdot$ & 0 & 1 & 2 & 3 & 4 & 5 & 6 & 7 \\
\hline 0 & 0 & 0 & 0 & 0 & 0 & 0 & 0 & 0 \\
1 & 0 & 4 & 4 & 0 & 0 & 4 & 4 & 0 \\
2 & 0 & 4 & 4 & 0 & 0 & 4 & 4 & 0 \\
3 & 0 & 0 & 0 & 0 & 0 & 0 & 0 & 0 \\
4 & 0 & 3 & 3 & 0 & 0 & 3 & 3 & 0 \\
5 & 0 & 7 & 7 & 0 & 0 & 7 & 7 & 0 \\
6 & 0 & 7 & 7 & 0 & 0 & 7 & 7 & 0 \\
7 & 0 & 3 & 3 & 0 & 0 & 3 & 3 & 0
\end{tabular}

Mace4 [2] suggests that this is indeed the example of smallest order of a nonassociative LA-ring. By checking the tables up to order 63 through Mace4, it further suggests that nonassociative LA-rings only exist of orders 8,9,12 and 18. Mace4 gives us the lower bound of the number of nonassociative LA-rings of some orders. For example by using isofilter of Mace4 there are at least 
192 nonisomorphic nonassociative LA-rings of order 8, at least 502 nonisomorphic nonassociative LA-rings of order 9 and at least 648 nonisomorphic nonassociative LA-rings of order 12 . *

However Mace4 exits at order 64 due to memory exhaustion. Thus we are unable to determine what is the next order after 18 for which nonassociative LA-ring exists. So this will be an interesting question to be settle down for future research.

Example 2. The following example shows the existence of an LA-ring with left identity of order 9, where

(i) is a nonassociative AG-group,

(ii) is a nonassociative AG-groupoid.

(i)

\begin{tabular}{|c|c|c|c|c|c|c|c|c|c|c|c|c|c|c|c|c|}
\hline+ & 0 & 1 & 23 & 4 & & & 7 & . & U & & & & & & & \\
\hline 0 & 3 & 4 & 68 & 7 & 2 & 51 & 10 & 0 & 3 & 1 & 6 & & & & & \\
\hline 1 & 2 & 3 & 76 & 8 & 4 & 10 & 05 & 1 & 0 & 3 & 0 & 3 & 3 & & & \\
\hline 2 & 1 & & 34 & 2 & 0 & 8 & 67 & 2 & 8 & 1 & 5 & & $\gamma$ & & & \\
\hline 3 & 0 & & 23 & 4 & 5 & & 78 & 3 & 3 & 3 & 3 & 3 & & & & \\
\hline 4 & 5 & 0 & 42 & 3 & 1 & 7 & 86 & 4 & 0 & 6 & 7 & 3 & 5 & & & \\
\hline 5 & 4 & & 87 & 6 & 3 & & $\begin{array}{ll}5 & 1\end{array}$ & 5 & 8 & 6 & 4 & 3 & 2 & 7 & & \\
\hline 6 & 7 & & $\begin{array}{ll}0 & 1\end{array}$ & 5 & 8 & & 24 & 6 & & 3 & 8 & & & & & \\
\hline 7 & 6 & 8 & 15 & 0 & 7 & & 32 & 7 & U & 1 & $Z$ & & & & & \\
\hline 0 & 8 & 7 & & 1 & 6 & 2 & 43 & 8 & 3 & 6 & & & & & & \\
\hline
\end{tabular}

Example 3. The following example shows the existence of an LA-ring with left identity of order 9, where

(i) is a nonassociative AG-group,

(ii) is a nonassociative AG-groupoid.

(i)

\begin{tabular}{|c|c|c|c|c|c|c|c|c|c|c|c|c|c|c|c|c|}
\hline$T$ & $\begin{array}{ll}0 & 1\end{array}$ & 12 & 3 & 4 & & & 8 & & 0 & & & & & & & \\
\hline 0 & 34 & 46 & 8 & 7 & 25 & 51 & 0 & 0 & 3 & 1 & 6 & $?$ & & 6 & 6 & \\
\hline 1 & 23 & 37 & 6 & 8 & $\begin{array}{ll}4 & 1\end{array}$ & 10 & 5 & 1 & 0 & 3 & 0 & 3 & 8 & 8 & 3 & \\
\hline 2 & 15 & 53 & 4 & 2 & 08 & 86 & 7 & 2 & 8 & 1 & 5 & 3 & 7 & & 6 & \\
\hline 3 & $\begin{array}{ll}0 & 1\end{array}$ & 12 & 3 & 4 & 56 & 67 & 8 & 3 & 3 & 3 & 3 & 3 & 3 & 3 & 3 & \\
\hline 4 & 50 & $\begin{array}{ll}04 \\
0\end{array}$ & 2 & 3 & 17 & 78 & 6 & 4 & 0 & 6 & 7 & 3 & 5 & 4 & 1 & \\
\hline 5 & 42 & 28 & 7 & 6 & 30 & 5 & 1 & 5 & 8 & 6 & 4 & 3 & 2 & 7 & 1 & \\
\hline 6 & 76 & 60 & 1 & 5 & 83 & 32 & 4 & 6 & & 3 & 8 & 3 & U & & 3 & \\
\hline 7 & 68 & $\begin{array}{ll}81 \\
8\end{array}$ & 5 & & 74 & 43 & 2 & 7 & & & & 3 & & & 0 & \\
\hline 0 & 87 & 75 & 0 & 1 & & 24 & 3 & 8 & . & 6 & 1 & 3 & & 1 & & \\
\hline
\end{tabular}

*All tables of LA-ring are available from the authors by an email request. 
Example 4. The following example shows the existence of an LA-field of order 9, where

(i) is a nonassociative AG-group,

(ii) is a nonassociative AG-groupoid.

(i)

\begin{tabular}{c|lllllllll}
+ & 0 & 1 & 2 & 3 & 4 & 5 & 6 & 7 & 8 \\
\hline 0 & 3 & 0 & 5 & 1 & 8 & 7 & 4 & 2 & 6 \\
1 & 1 & 3 & 7 & 0 & 6 & 2 & 8 & 5 & 4 \\
2 & 4 & 6 & 3 & 8 & 7 & 1 & 5 & 0 & 2 \\
3 & 0 & 1 & 2 & 3 & 4 & 5 & 6 & 7 & 8 \\
4 & 2 & 7 & 6 & 5 & 3 & 4 & 0 & 8 & 1 \\
5 & 6 & 8 & 0 & 4 & 5 & 3 & 2 & 1 & 7 \\
6 & 5 & 2 & 4 & 7 & 1 & 8 & 3 & 6 & 0 \\
7 & 8 & 4 & 1 & 6 & 2 & 0 & 7 & 3 & 5 \\
8 & 7 & 5 & 8 & 2 & 0 & 6 & 1 & 4 & 3
\end{tabular}

(ii)

\begin{tabular}{c|lllllllll}
$\cdot$ & 0 & 1 & 2 & 3 & 4 & 5 & 6 & 7 & 8 \\
\hline 0 & 4 & 5 & 1 & 3 & 6 & 7 & 2 & 8 & 0 \\
1 & 5 & 4 & 0 & 3 & 7 & 6 & 8 & 2 & 1 \\
2 & 6 & 7 & 5 & 3 & 2 & 8 & 1 & 0 & 4 \\
3 & 3 & 3 & 3 & 3 & 3 & 3 & 3 & 3 & 3 \\
4 & 1 & 0 & 8 & 3 & 5 & 4 & 7 & 6 & 2 \\
5 & 0 & 1 & 2 & 3 & 4 & 5 & 6 & 7 & 8 \\
6 & 8 & 2 & 6 & 3 & 0 & 1 & 4 & 5 & 7 \\
7 & 2 & 8 & 7 & 3 & 1 & 0 & 5 & 4 & 6 \\
8 & 7 & 6 & 4 & 3 & 8 & 2 & 0 & 1 & 5
\end{tabular}

The LA-ring $(R,+,$.$) given in the above example is indeed an LA-field as$ $(R,+)$ is a nonassociative AG-group having 3 as a left identity and $(R-\{3\},$. is also a nonassociative AG-group having 5 as a left identity and both distributive laws hold. For the fact that $(R-\{3\},$.$) is a nonassociative AG-group with$ left identity 5 indeed, we have used GAP [1].

Remark 1. By [5, Corollary 4] in an LA-field $(F,+,$.$) the additive AG-$ group $(F,+)$ will always has all elements as self-inverse. Note that this is not necessary for an AG-group if it is not an LA-field as many examples of such an AG-group can be found in [3, 4].

Associativity in the multiplicative AG-groupoid of an LA-ring does not force associativity in the additive AG-group even if it be an LA-field. Consider the following example of an LA-field $(F,+,$.$) of order 3$ in which $(F-\{0\},$. is an abelian group yet $(F,+)$ is nonassociative.

Example 5. The following example shows the existence of an LA-field of order 3, where

(i) is a nonassociative AG-group,

(ii) is a commutative semigroup and hence is an AG-groupoid.

\begin{tabular}{|c|c|c|c|c|c|}
\hline \multicolumn{3}{|c|}{ (i) } & \multicolumn{3}{|c|}{ (ii) } \\
\hline+ & & 12 & & & 12 \\
\hline 0 & 0 & 12 & $\overline{0}$ & & 00 \\
\hline 1 & & $\begin{array}{ll}0 & 1\end{array}$ & 1 & 0 & \\
\hline 2 & 1 & 20 & 2 & & $\begin{array}{ll}21 \\
\end{array}$ \\
\hline
\end{tabular}


Note that associativity of addition is not allowed in LA-ring. If we allow it we can see that the converse of Example 5 is also true, that is, associativity in the additive AG-group of an LA-ring does not force associativity in the multiplicative AG-groupoid. By doing so we get a sort of special LA-ring and thus we call it a Special LA-ring. Let us define it properly.

Definition 1. A Special LA-ring is a nonassociative structure having at least two binary operations ' + ' and '.'such that $(R,+)$ is an abelian group and $(R,$. is an AG-groupoid (LA-semigroup) and the two distributive laws also hold in $R$.

Recall from [3] that associativity and commutativity are equivalent for an AG-group.

Example 6. The following example shows the existence of a nonassociative Special LA-ring of order 8, where

(i) is an abelian group,

(ii) is a nonassociative AG-groupoid.

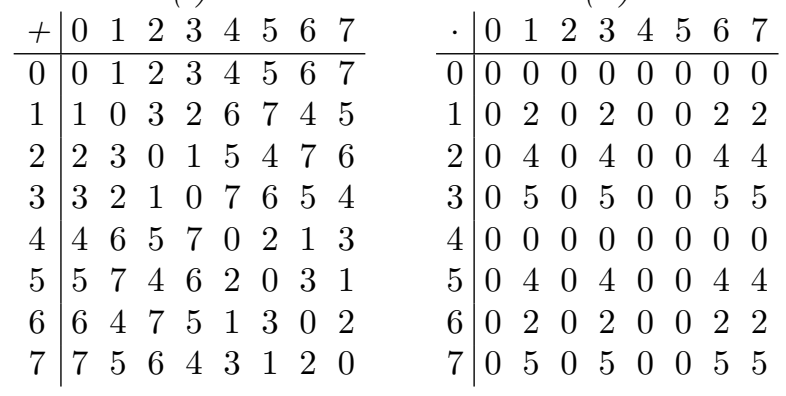

Open problem: Finally the LA-ring given in Example $3,(R,$.$) has left$ identity 7 while $(R,+)$ has left identity 3 . If we consider $(R-\{3\},$.$) , then$ this is not an AG-groupoid because it is not closed as for example $6 \cdot 1=3$ that does not belong to $R-\{3\}$. It can happen to be an AG-groupoid as in Examples 3 and 4 . Suppose this is the case then having left identity it becomes an AG-monoid (An AG-groupoid with left identity is called an AGmonoid). Now whether there exists an example in which it remains merely an AG-monoid and not an AG-group, we are unable to settle it. So we leave it as open problem. However we give the following conjecture.

Conjecture 1. Let $(R,+,$.$) be an LA-ring having multiplicative left identity$ $e$ and additive left identity 0 . If $(R-\{0\},$.$) is an A G$-monoid, then it will be an AG-group. 


\section{References}

[1] GAP - Groups, Algorithms and Programming. Version 4.4.12. 2008. http://www.gap-system.org

[2] McCune W. Prover9 and MACE4. http://www.cs.unm.edu/mccune/mace4/

[3] Shah M, Ali A. Some structural properties of AG-group. Int. Math. Forum, 2011, 6(34): 1661-1667

[4] Shah M, Gretton C, Sorge V. Enumerating AG-groups with an introduction to Samarandache AG-groups. Int. Math. Forum 2011, 6(62): 30793086

[5] Shah M, Shah T. Some basic properties of LA-rings. Int. Math. Forum, 2011, 6(44): 2195-2199

[6] Shah T, Rehman I. On LA-Rings of finite nonzero functions. Int. J. Contemp. Math. Sciences, 2010, 5(5): 209-222

[7] Shah T, Raees M, Ali G. On LA-Modules. Int. J. Contemp. Math. Sciences, 2011, 6(21): 999-1006

[8] Shah T, Rehman I. On Characterization of LA-ring through some properties of their ideals. Southeast Asian Bulletin of Mathematics, to appear.

[9] Shah T, Kausar N, Rehman I. Intuitionistics fuzzy normal subrings over a nonassociative ring. Analele Stiintifice ale Universitatii Ovidius Constanta, 2012, 20: 369-386.

Inayatur-Rehman,

Department of Mathematics,

Comsats, Institute Of Information Technology,

Abbottabad, Pakistan.

Email: sinayat@ciit.net.pk

Muhammad Shah,

Department of Mathematics,

Abdul Wali Khan University,

KPK, Pakistan.

Email: shahmaths_problem@hotmail.com

Tariq Shah, Asima Razzaque,

Department of Mathematics,

Quaid-i-Azam University,

Islamabad, Pakistan.

Email: stariqshah@gmail.com

Email: asimarazzaque@ue.edu.pk 